\title{
Chloroma/Granulocytic Sarcoma: Abdominal \& Pelvic Presentation of Acute Myelogenous Leukemia
}

\author{
Vassiliki Liana Tsikitis ${ }^{\bowtie}$, Cybil Corning, Jeff Henderson, Jessica Rose \\ Department of Surgery of College of Medicine, University of Arizona, Tucson, AZ 85724-5131, USA
}

$\triangle$ Corresponding author: Vassiliki Liana Tsikitis, MD, 1501 N. Campbell Avenue; PO Box 245131; Tucson, AZ 85724-5131. (520) 626-6788 office/(520) 626-7785 fax; Email: ltsikitis@surgery.arizona.edu

Published: 2010.07.14

\begin{abstract}
There is limited literature documenting granulocytic sarcoma of the colon. We report a case of a 28 year-old female with a colonic granulocystic sarcoma of the colon as a complication of $A M L$, as it is an important consideration with surgical management of typhilitis.
\end{abstract}

Key words: Granulocytic Sarcoma, Acute Myelogenous Leukemia, Peritonitis, Colon

\section{Case Report}

A 28-year-old woman with a history of acute myelogenous leukemia (AML), aggressively treated with 3 bone marrow transplants and multiple regimens of chemotherapy targeting AML, presented to the emergency room with abdominal pain and fever. Computed tomography (CT) of the abdomen and pelvis revealed pneumatosis of the transverse colon and a small amount of free air adjacent to the lesser sac (Figure 1).

The patient's history of AML was significant for extensive tumor burden and uterine involvement, causing hydronephrosis and renal failure, despite her multiple chemotherapy regimens. Additionally, she had previously undergone an ileocecectomy for typhilitis.

Our surgical examination showed the patient to be alert, afebrile, and hemodynamically stable, with mild tenderness on palpation of the abdomen. Our laboratory examination revealed a white blood cell count of 5.4; hemoglobin, 9.3; and platelets, 38. Of note, a bone marrow biopsy performed 1 week earlier showed hypocellular marrow, without morphologic or immunophenotypic evidence of acute leukemia.

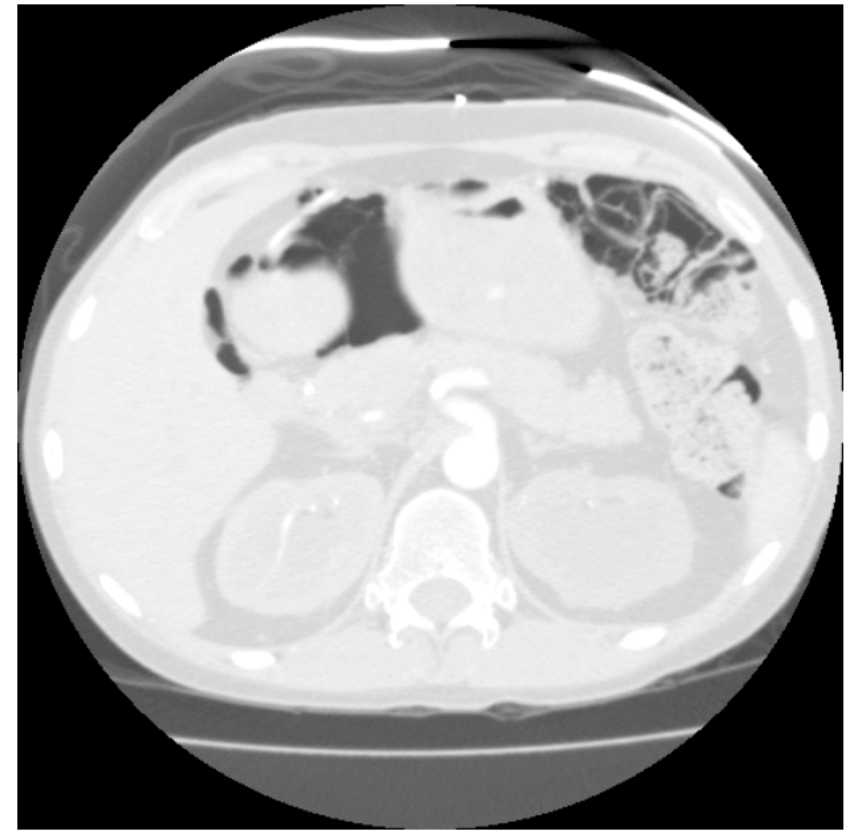

Figure I. Computed tomography (CT) of the abdomen and pelvis revealed pneumatosis of the transverse colon and a small amount of free air adjacent to the lesser sac. 
We performed an exploratory laparotomy. We noted diffuse pneumatosis and thickened, ischemic-appearing transverse colon extending to the splenic flexure. We then performed an extended right colectomy, including the remaining right colon and transverse colon, with a long Hartmann pouch and end ileostomy. She tolerated the operation well and was extubated postoperatively without immediate complications.

Our pathologic examination demonstrated extensive pneumatosis intestinalis (Figure 2, hematoxylin and eosin [H\&E], 40X), and a focus of myeloid (granulocytic) sarcoma at the ileal resection margin. The ileal resection margin contained a small $(0.9 \mathrm{~cm} \mathrm{x}$ $0.5 \mathrm{~cm}$ ) serosal nodule composed of an infiltrate of granulocytic precursor cells. Those cells ranged from the most immature blast forms to mature neutrophils - a spectrum consistent with granulocytic sarcoma (also called myeloid sarcoma or chloroma). On the serosal surface, cells formed a discrete mass; they did not involve either the muscular wall or the mucosa of the small bowel. Immuno-histochemistry testing of the mass stained positively for the immature myeloid markers CD 34 (Figure 3, 40X) and CD 117 (Figure 4, 40X); the pattern matched the patient's original diagnostic bone marrow biopsy findings for AML from 3 $1 / 2$ years previously. This tissue manifestation of AML represented a relapse of the patient's original disease process.

Granulocytic sarcoma is a malignant tumor found in a number of extramedullary sites. Frequently, it is found concurrently with AML, but can also be found as an isolated lesion in patients who do not have AML. Extramedullary sites include the skin, lymph nodes, central nervous system, reproductive organs, and gastrointestinal (GI) tract. $(1-5,9,10)$.

Our patient represents an unusual complication of AML, namely, granulocytic sarcoma in multiple extramedullary sites, including the uterus, vagina, and colon. Most reported cases of granulocytic sarcoma $(69 \%)$ are reported in patients with a single focus of disease or an isolated mass (2). In up to $36 \%$ of women with AML, autopsy shows genital tract involvement (4), but only a handful of case reports have documented antemortem diagnosis $(7,8)$. Reports in the literature documenting the involvement of the colon are uncommon; and even more rare when additional sites are included. In the GI tract, the small bowel is more frequently involved than the colon; rates of small bowel involvement are estimated at $10 \%$ $(1,2)$. Large bowel involvement is evident primarily in case reports $(6,9,10)$.

When planning surgery for neutropenic patients with typhilitis, particularly those with AML, granu- locytic sarcoma should be considered in the differential diagnosis. Involvement of the colon may be more frequent than reported, given that typhilitis may be treated without surgery in a select population of patients. Moreover, involvement of the colon may be mistaken for more routine pathologic findings of colonoscopic evaluations or may be misdiagnosed by histologic tests $(3,6)$. The prognosis for patients with AML is grim. Multiple site involvement perhaps defines an advanced stage of the disease.

Our patient's recovery during the immediate postoperative period was uneventful. However, a month after surgery, she was readmitted for fever, neutropenia, and pneumonia -- which resulted in her death about 1 month later (that is, 2 months postoperatively).

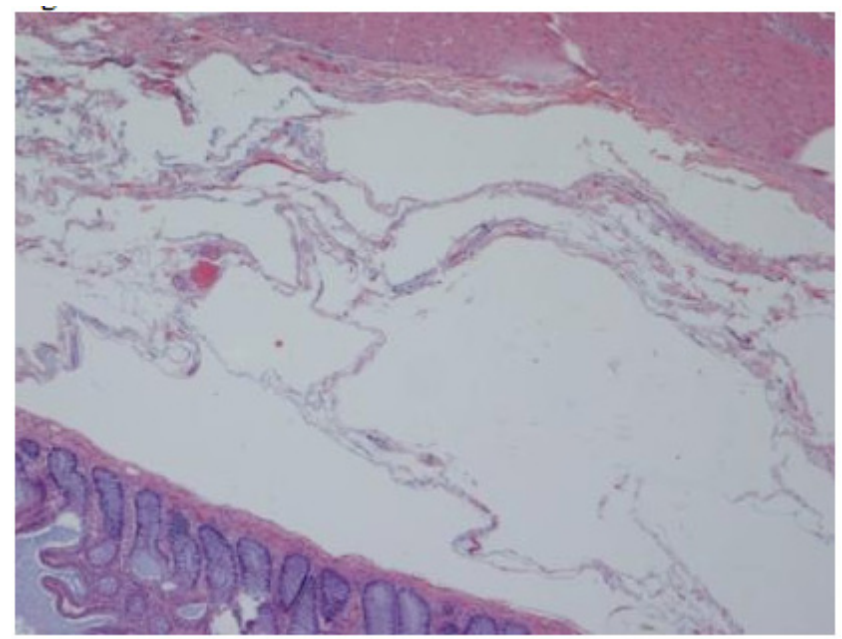

Figure 2. Hematoxylin and eosin [H\&E], 40X.

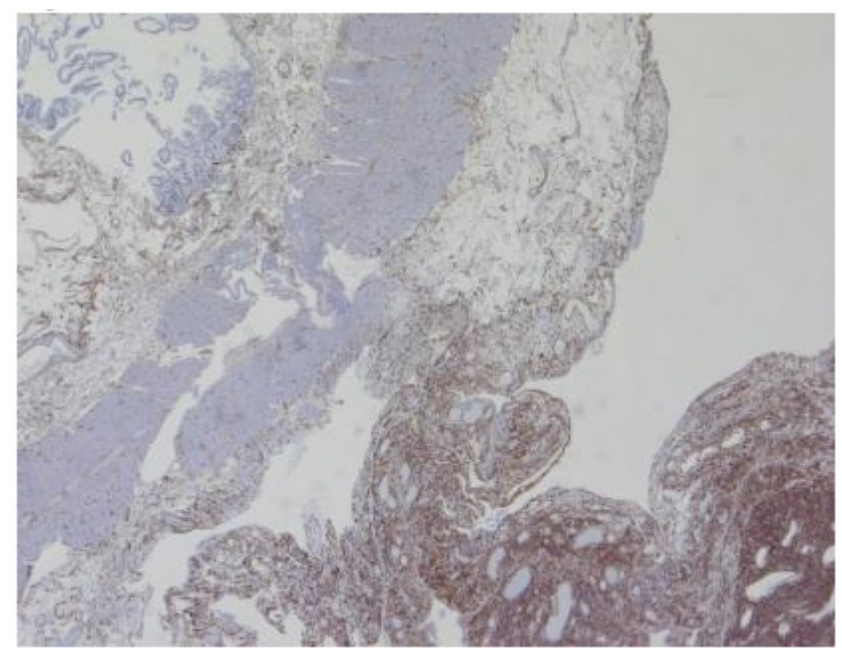

Figure 3. Immuno-histochemistry testing of the mass stained positively for the immature myeloid marker CD 34 (40X). 


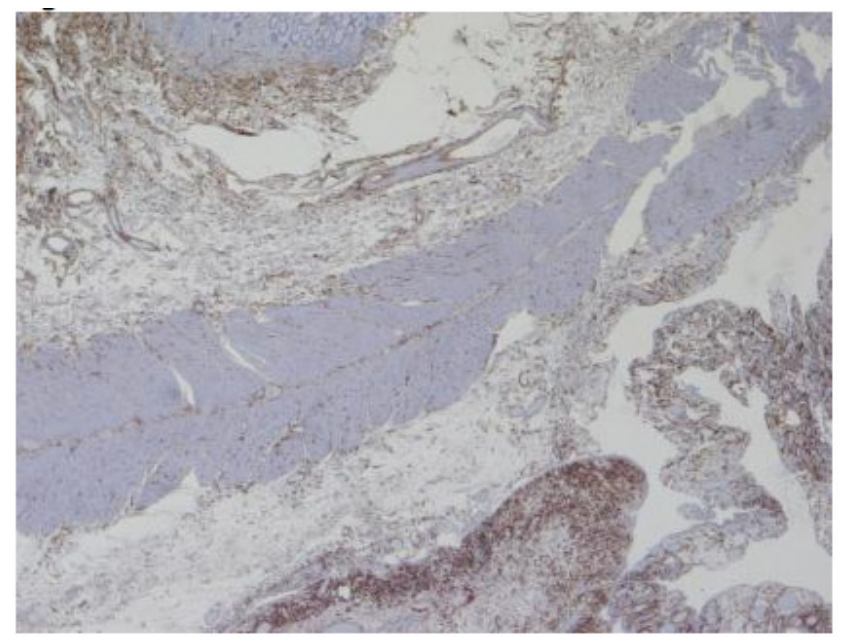

Figure 4. Immuno-histochemistry testing of the mass stained positively for the immature myeloid marker CD II7 (40X).

\section{Conflict of Interest}

The authors have declared that no conflict of interest exists.

\section{References}

1. Kitagawa Y, Sameshima Y, Shiozaki H, et al. Isolated graulocytic sarcoma of the small intestine successfully treated with chemotherapy and bone marrow transplantation. Int J Hematol. 2008; 87: 410-3.

2. Kohl SK, Aoun K. Granulocytic sarcoma of the small intestine. Arch Pathol Lab Med. 2006; 130: 1570-4.

3. Lee SY, Park SJ, Kim YH, Lee JH. Nonleukemic granulocytic sarcoma presenting as intussusception of small bowel. Int J Clin Oncol. 2008; 13: 467-70.

4. Oliva E, Ferry J, Young RH, Prat J, Srigley J, Scully RE. Granulocytic sarcoma of the female genital tract: A clinicopathologic study of 11 cases. Am J Surg Pathol. 1997; 21: 1156-65.

5. Toki H, Okabe K, Kimura Y, et al. Granulocytic sarcoma of the intestine as a primary manifestation nine months prior to overt myelogenous leukemia. Jpn J Clin Oncol. 1987; 12: 79-85.

6. Gorczyca W, Weisberger J, Seiter K. Colonic adenomas with extramedullary myeloid tumor (granulocytic sarcoma). Leukemia and Lymphoma. 1999; 34: 621-4.

7. Maeng H, Cheong JW, Lee ST, et al. Isolated extramedullary relapse of acute myelogenous leukemia as a uterine granulocytic sarcoma in an allogeneic hematopoietic stem cell transplantation recipient. Yonsei Medical Journal. 2004; 45: 330-3.

8. Pathak B, Bruchim I, Brisson ML, et al. Granulocytic sarcoma presenting as tumors of the cervix. Gynecologic Onocology. 2005; 98: 493-7.

9. Catalano MF, Levin B, Hart RS, et al. Granulocytic sarcoma of the colon. Gastroenterology. 1991; 100: 555-9.

10. Byrd JC, Edenfield WJ, Shields DJ, Dawson NA. Extramedullary myeloid cell tumors in acute nonlymphocytic leukemia: a clinical review. J Clin Oncol. 1995; 13: 1800-16.

11. Tummala M, Thomas L, Gocke C, et al. Granulocytic sarcoma of the colon. J Clin Oncol. 2005; 128: 5840-1. 